$T I D / S N M--22$

\title{
S10P4 \\ SYSTEM PERFORMANCE
}




\section{DISCLAIMER}

This report was prepared as an account of work sponsored by an agency of the United States Government. Neither the United States Government nor any agency Thereof, nor any of their employees, makes any warranty, express or implied, or assumes any legal liability or responsibility for the accuracy, completeness, or usefulness of any information, apparatus, product, or process disclosed, or represents that its use would not infringe privately owned rights. Reference herein to any specific commercial product, process, or service by trade name, trademark, manufacturer, or otherwise does not necessarily constitute or imply its endorsement, recommendation, or favoring by the United States Government or any agency thereof. The views and opinions of authors expressed herein do not necessarily state or reflect those of the United States Government or any agency thereof. 


\section{DISCLAIMER}

Portions of this document may be illegible in electronic image products. Images are produced from the best available original document. 


\section{TABLE OF CONTENTS}

Figure 1. System S10P4 Performance

Power vs. Load Resistance in $40^{\circ} \mathrm{F}$ Water

Figure 2. System S10P4 Performance

Voltage vs. Load Resistance in $40^{\circ} \mathrm{F}$ Water

Figure 3. System S10P4 Performance

Current vs. Load Resistance in $40^{\circ} \mathrm{F}$ Water

Figure 4. System S10P4 Performance

Power vs. Load Resistance in $60^{\circ} \mathrm{F}$ Water

Figure 5. System S10P4 Performance

Voltage vs. Load Resistance in $60 \mathrm{~F}$ Water

Figure 6. System S10P4 Performance

Current vs. Load Resistance in $60^{\circ} \mathrm{F}$ Water

Figure 7. System S10P4 Performance

Power vs. Load Resistance in $80^{\circ} \mathrm{F}$ Water

Figure 8. System S10P4 Performance

Voltage vs. Load Resistance in $80^{\circ} \mathrm{F}$ Water

Figure 9. System S10P4 Performance

Current vs. Load Resistance in $80^{\circ} \mathrm{F}$ Water

Figure 10. System S10P4 Performance

System Power, Voltage and Current vs. Water Temperature

Figure 11. System S10P4 Performance

TEG Power, Voltage and Current vs. Water Temperature

Figure 12. System S10P4 Performance

System Temperatures vs. Water Temperatures

Figure 13. System S10P4 Performance

System Temperatures vs. Water Temperatures

Figure 14. System S10P4 Performance

System Temperatures vs. Water Temperatures 


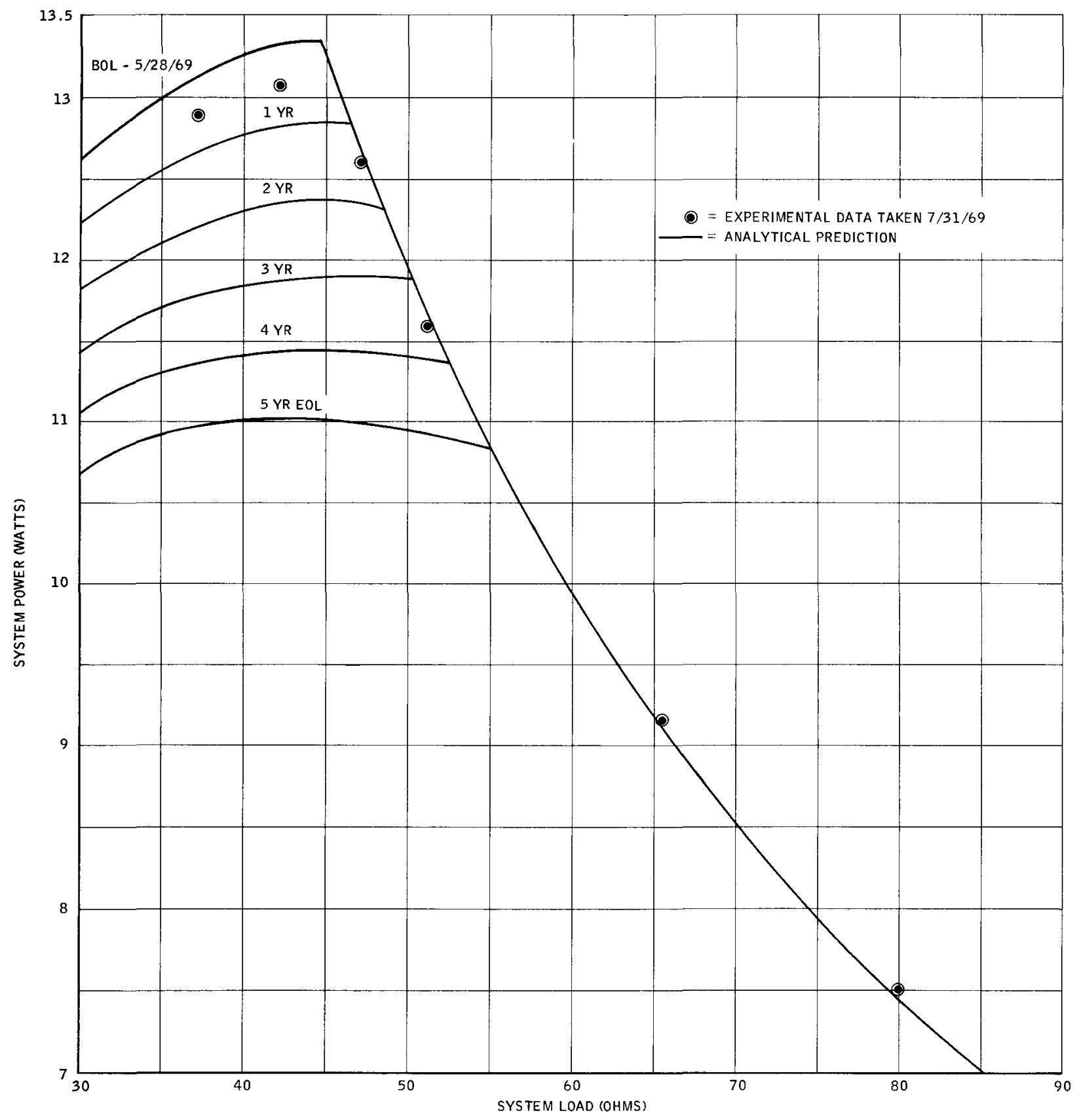

Figure 1. System S10P4 Performance

Power vs. Load Resistance in $40^{\circ} \mathrm{F}$ Water 


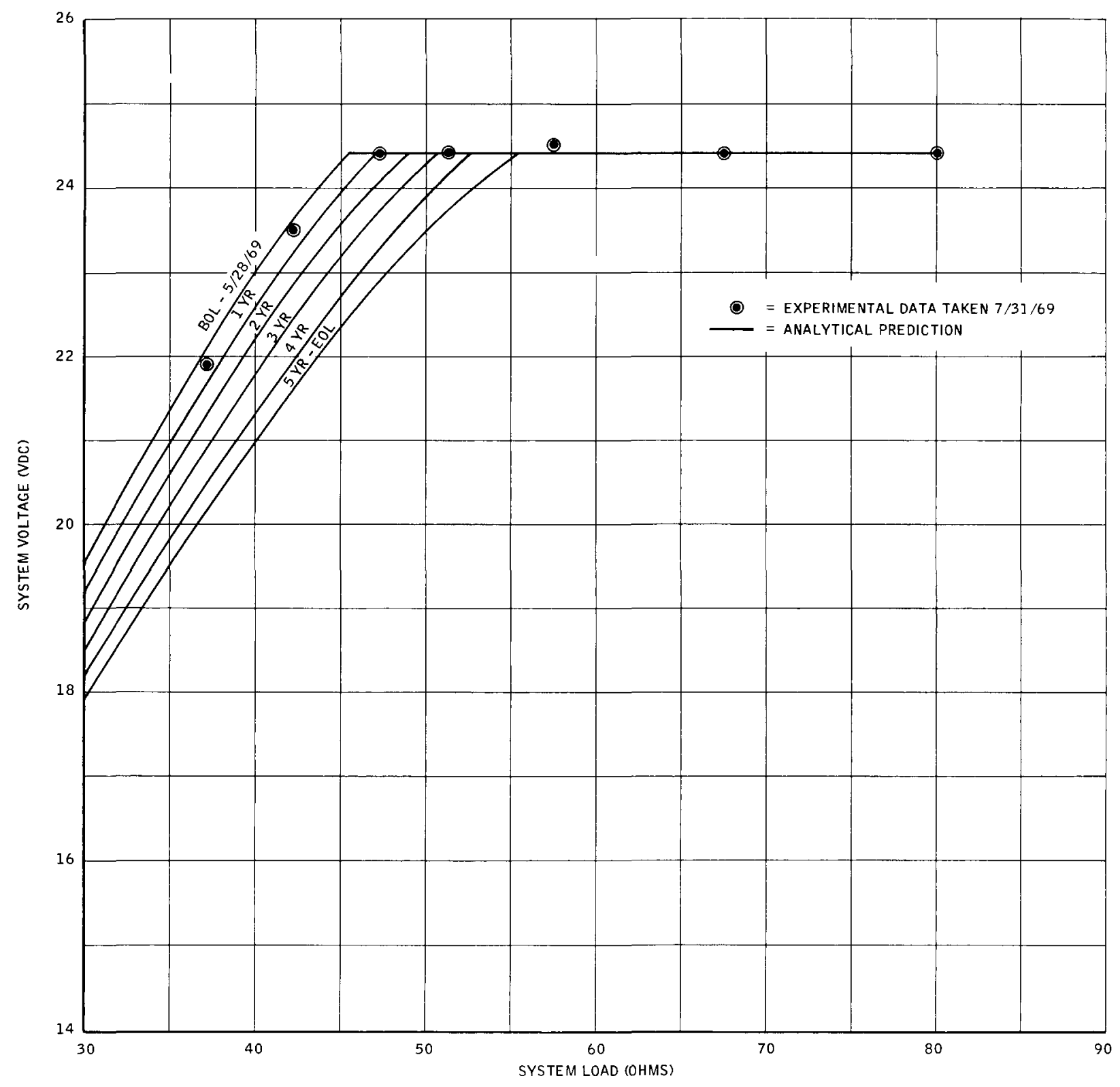

Figure 2. System S10P4 Performance

Voltage vs. Load Resistance in $40^{\circ} \mathrm{F}$ Water 


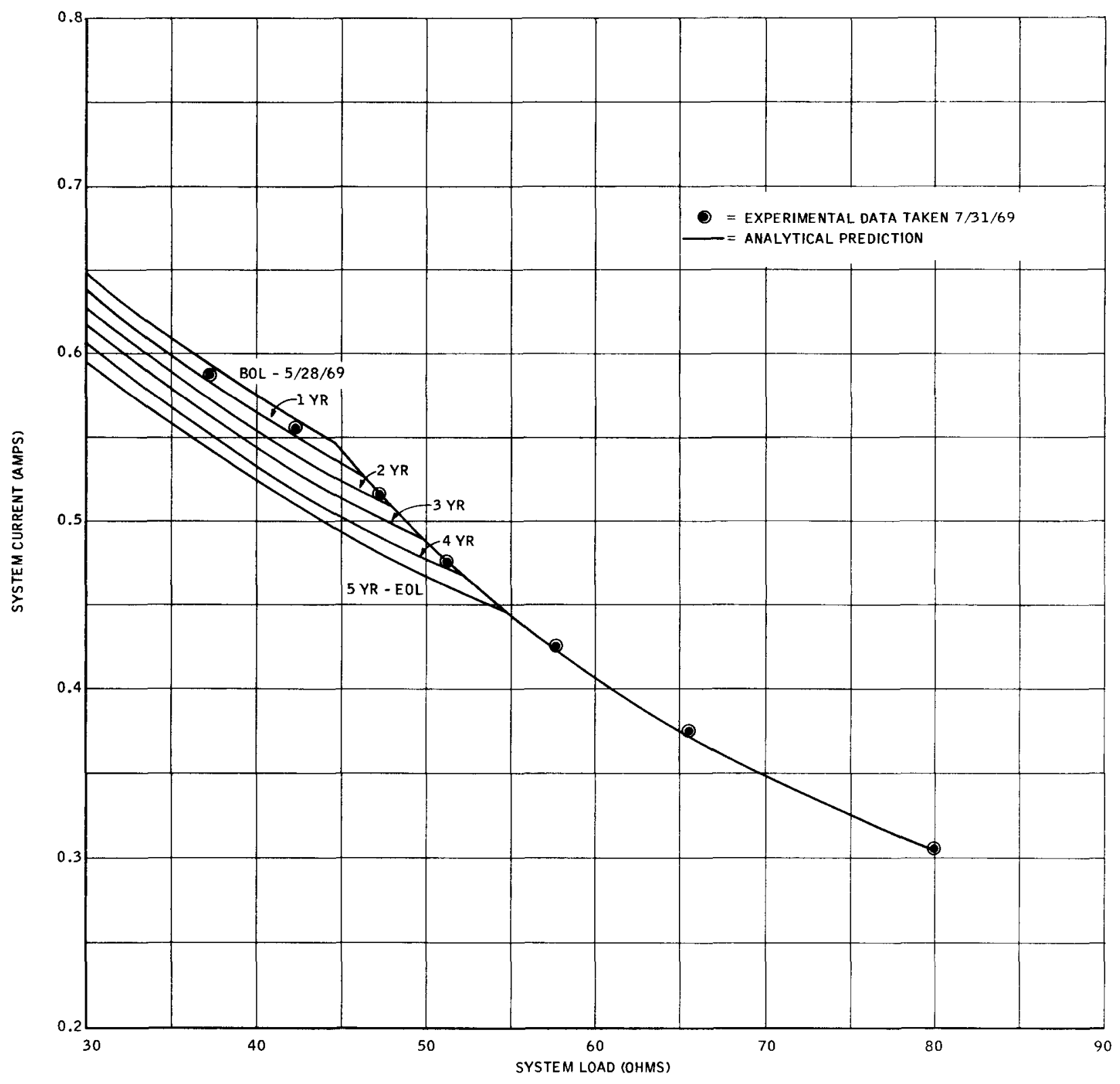

Figure 3. System S10P4 Performance

Current vs. Load Resistance in $40^{\circ} \mathrm{F}$ Water 


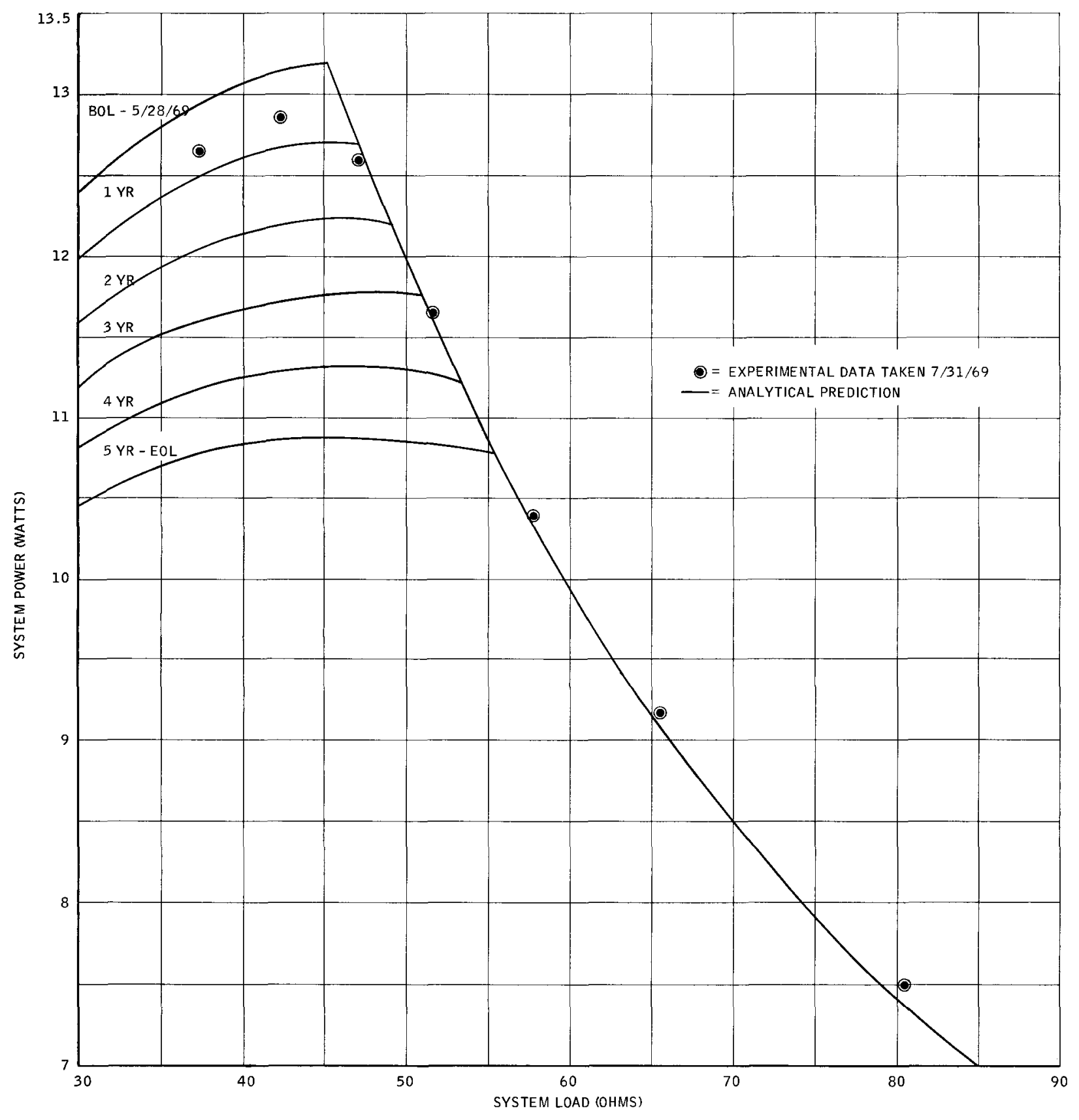

Figure 4. System S10P4 Performance

Power vs. Load Resistance in $60^{\circ} \mathrm{F}$ Water 


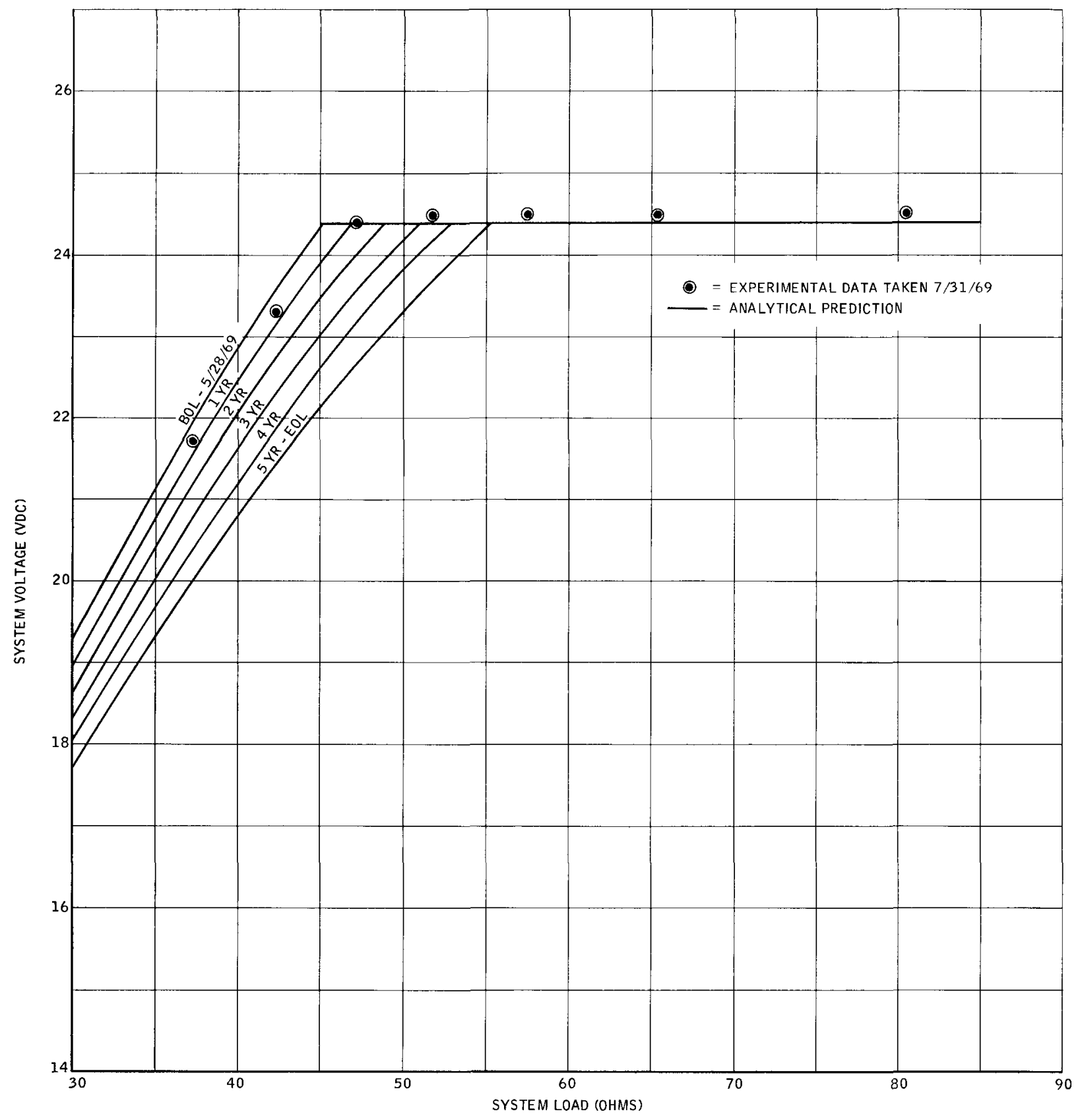

Figure 5. System S10P4 Performance

Voltage vs. Load Resistance in $60^{\circ} \mathrm{F}$ Water 


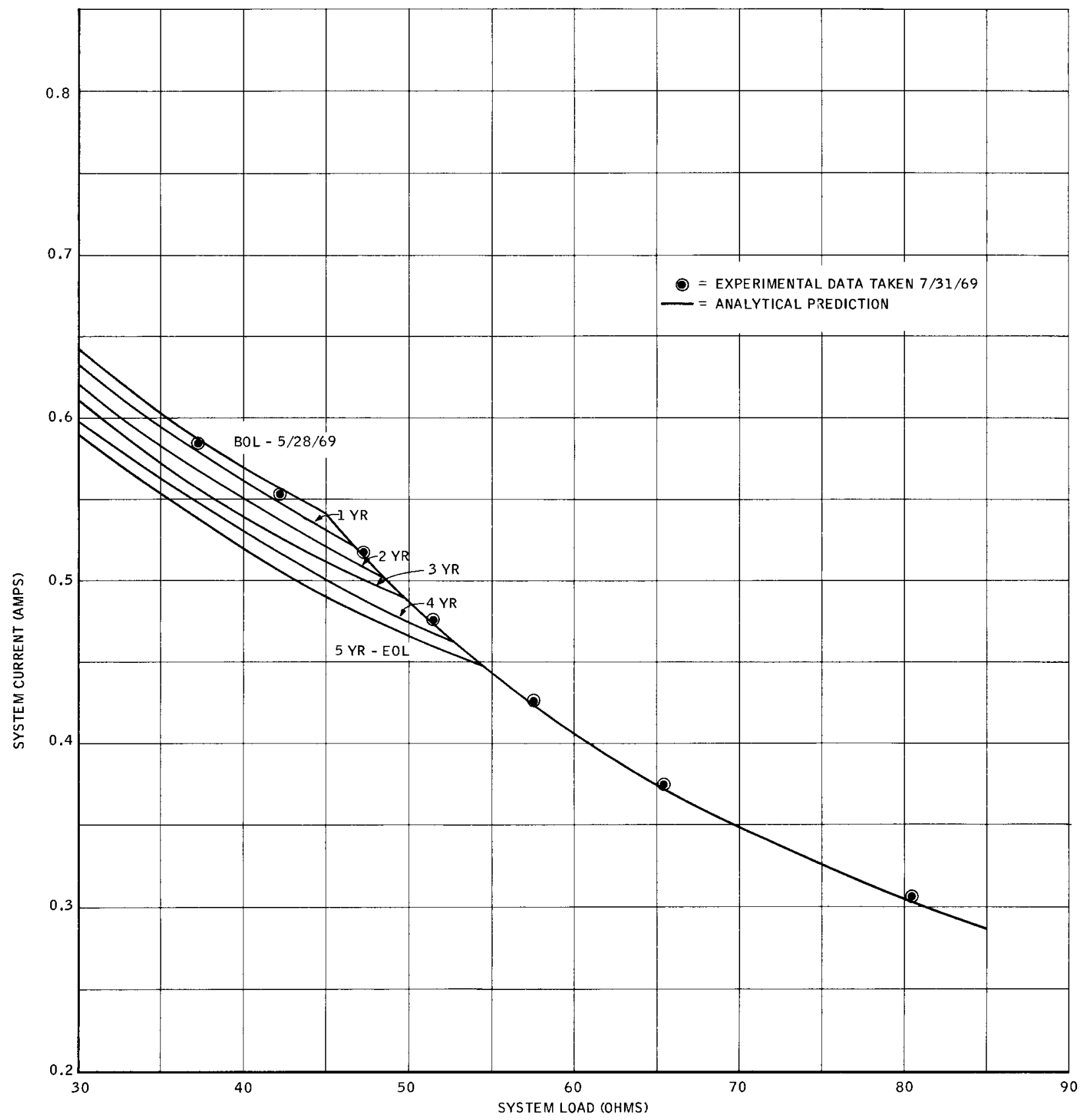

Figure 6. System S10P4 Performance

Current vs. Load Resistance in $60^{\circ} \mathrm{F}$ Water 


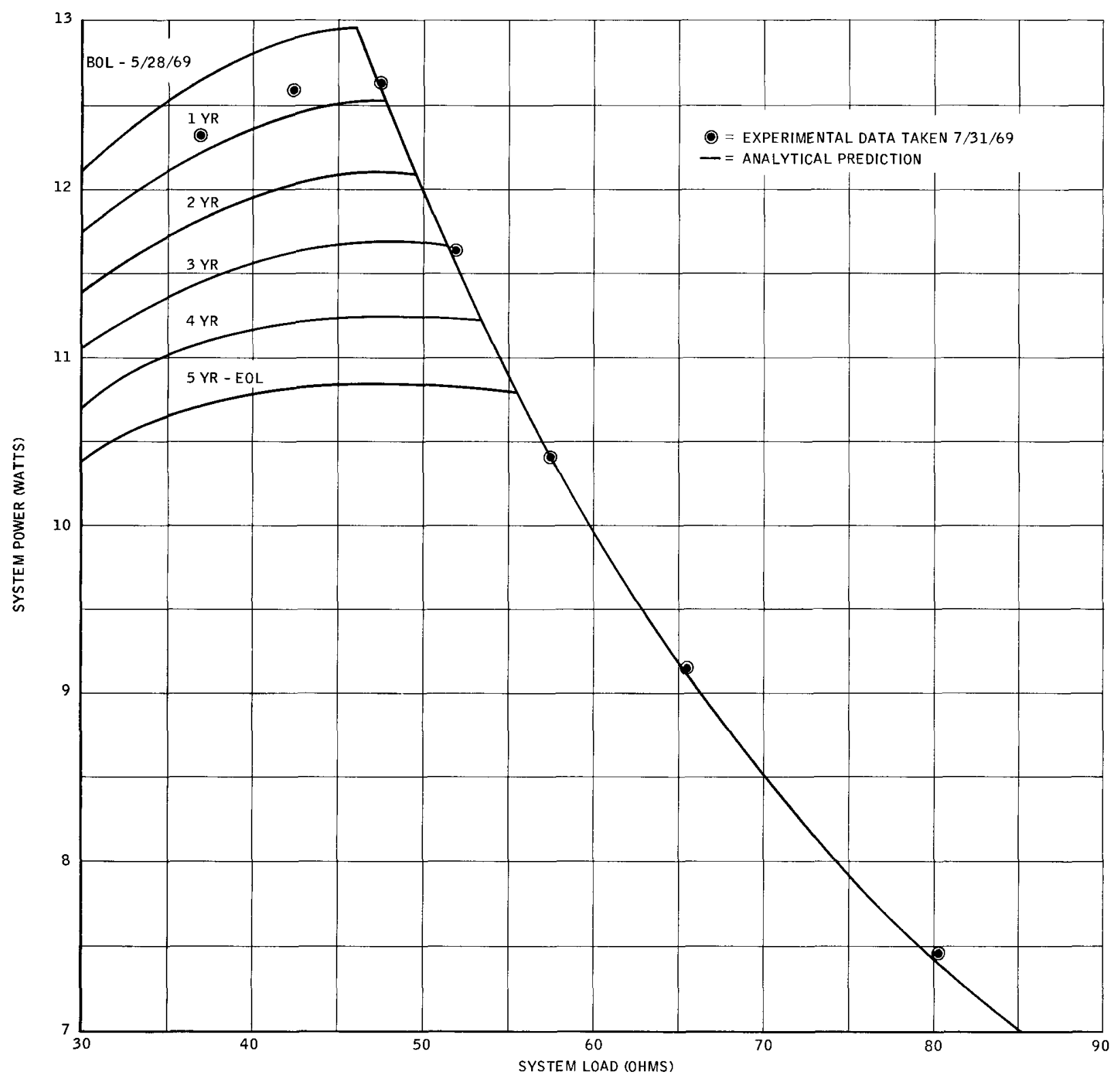

Figure 7. System S10P4 Performance

Power vs. Load Resistance in $80^{\circ} \mathrm{F}$ Water 


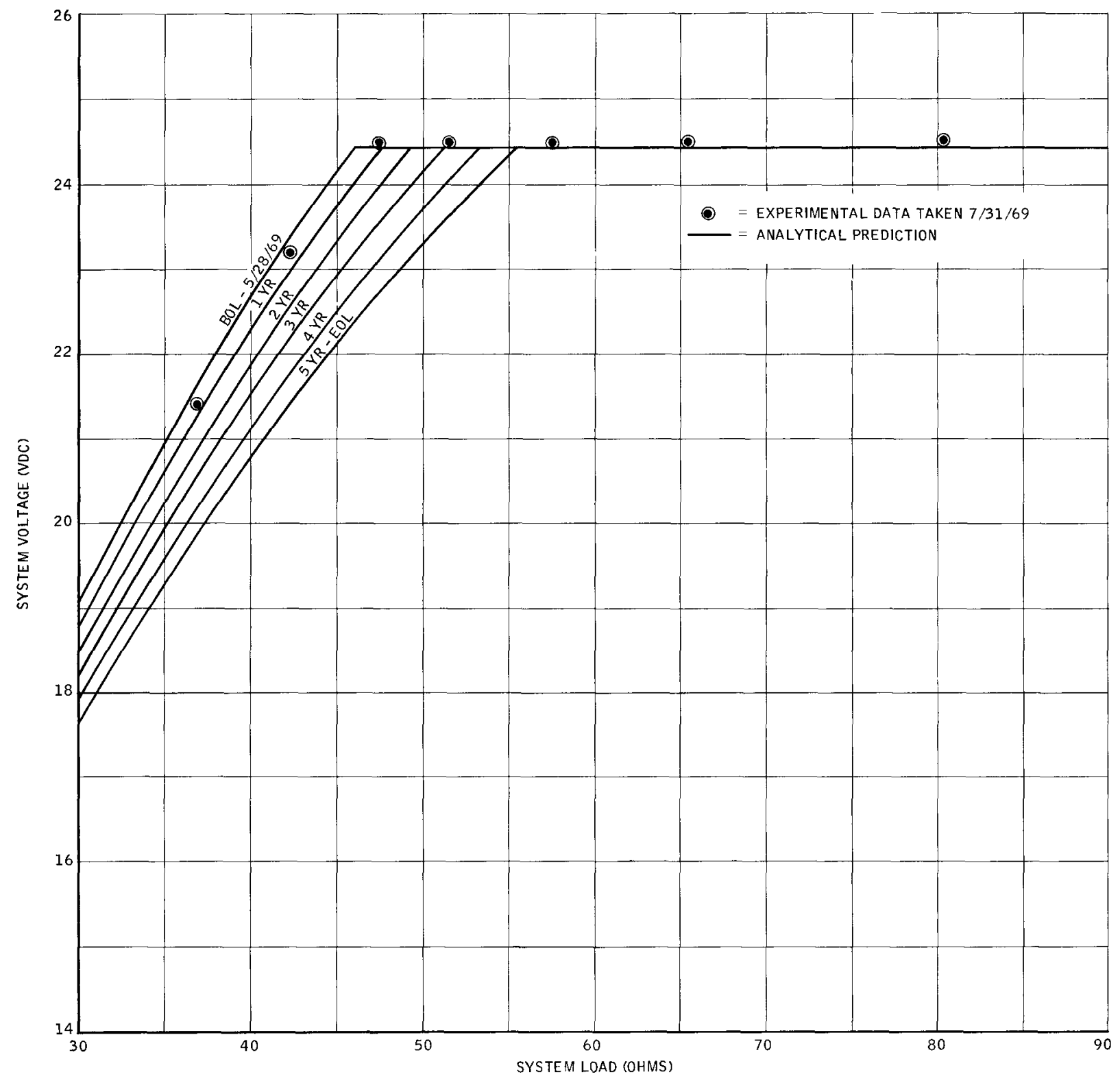

Figure 8. System S10P4 Performance

Voltage vs. Load Resistance in $80^{\circ} \mathrm{F}$ Water 


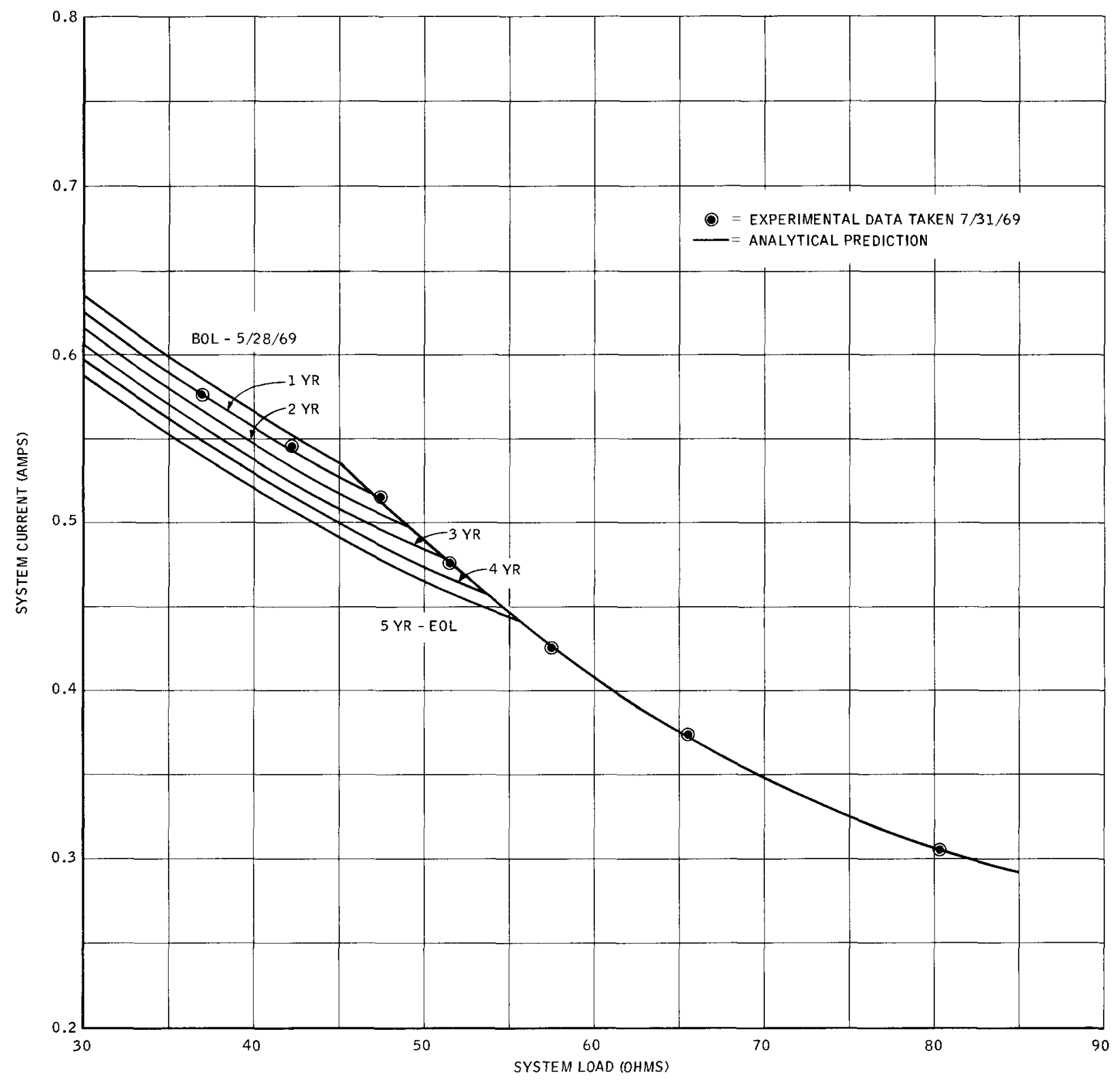

Figure 9. System S10P4 Performance

Current vs. Load Resistance in $80^{\circ} \mathrm{F}$ Water 


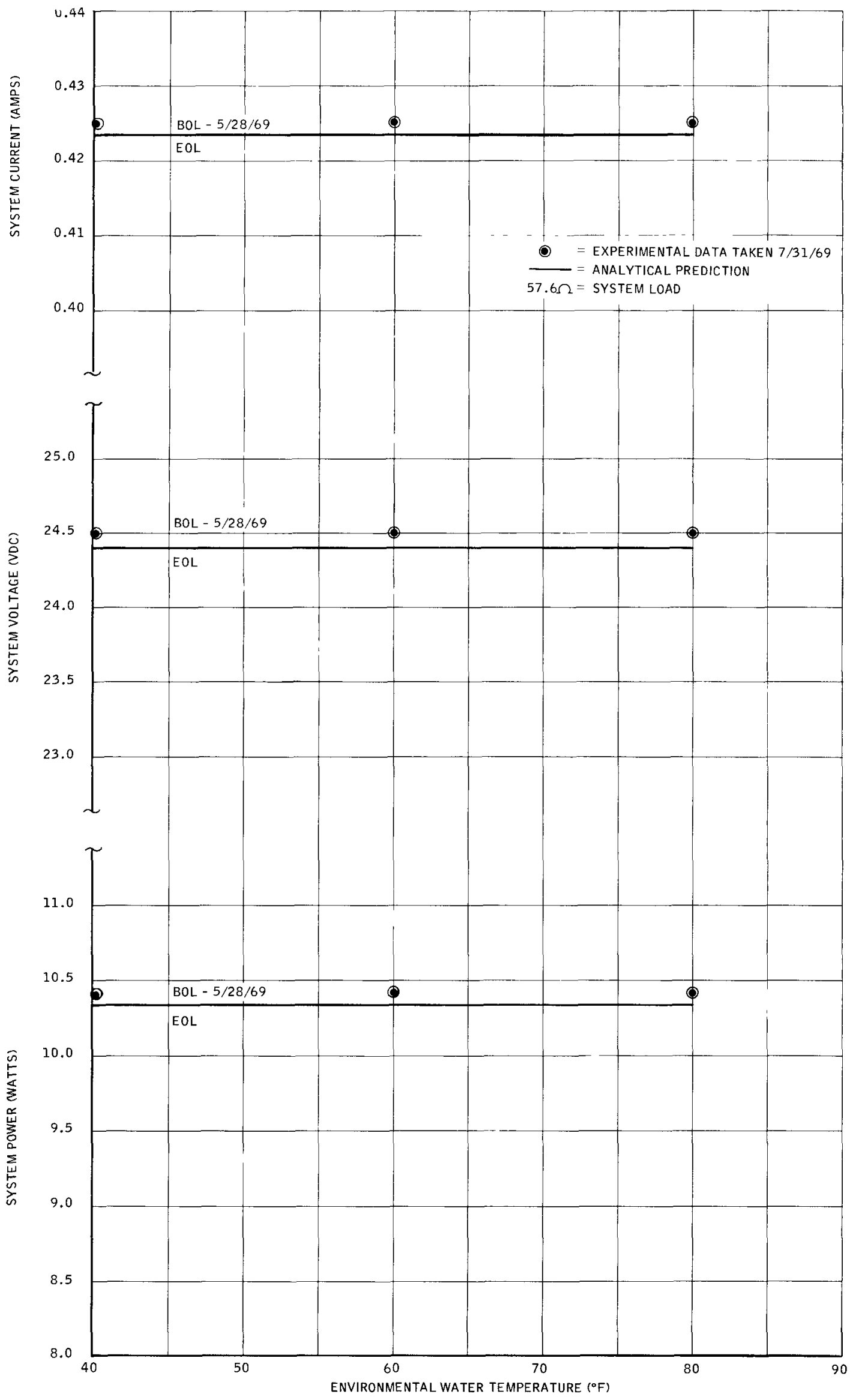

Figure 10. System S10P4 Performance System Power, Voltage and Current vs. Water Temperature 


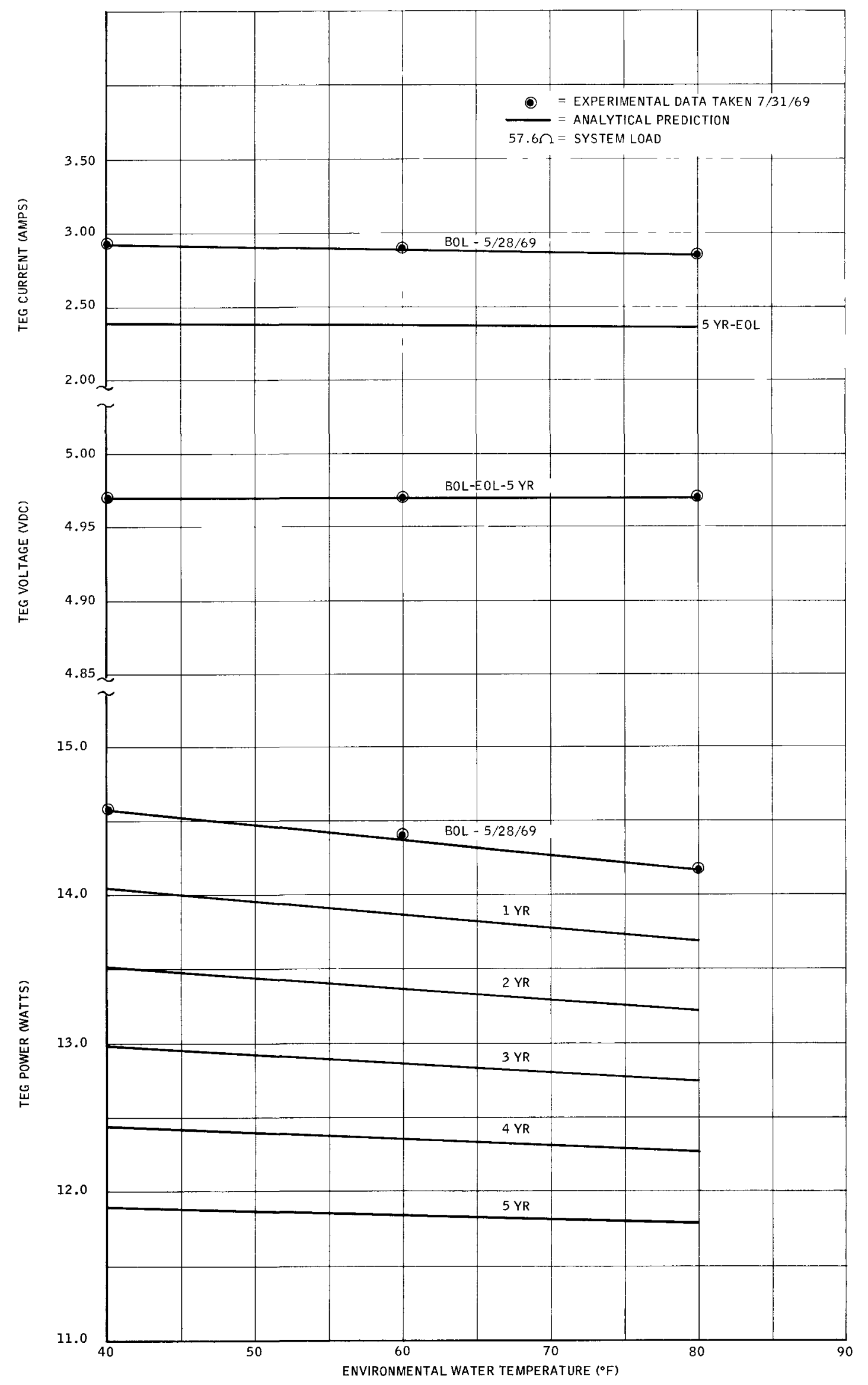

Figure 11. System S10P4 Performance TEG Power, Voltage and Current vs. Water Temperature 


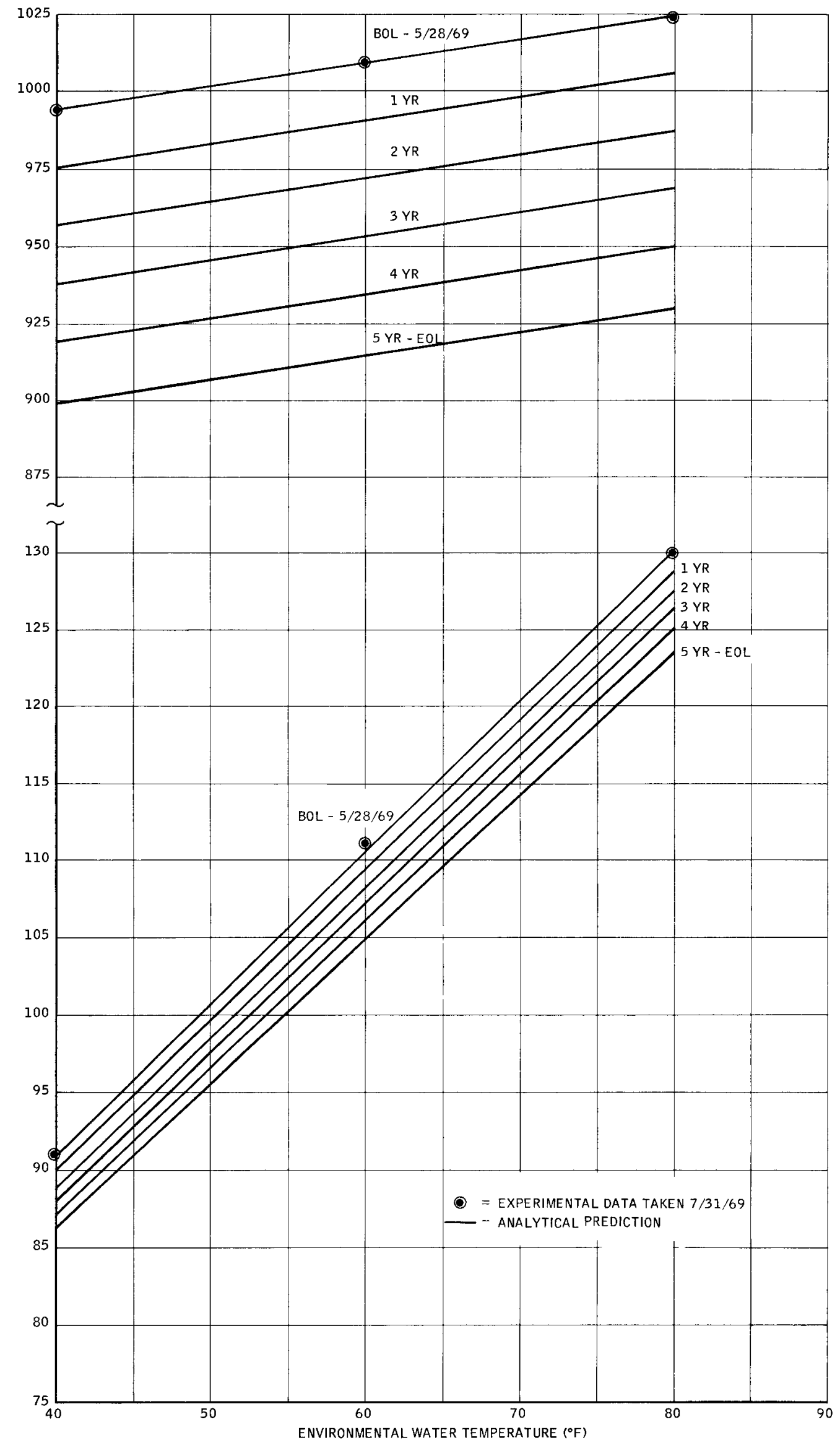

Figure 12. System S10P4 Performance

System Temperatures vs. Water Temperatures 


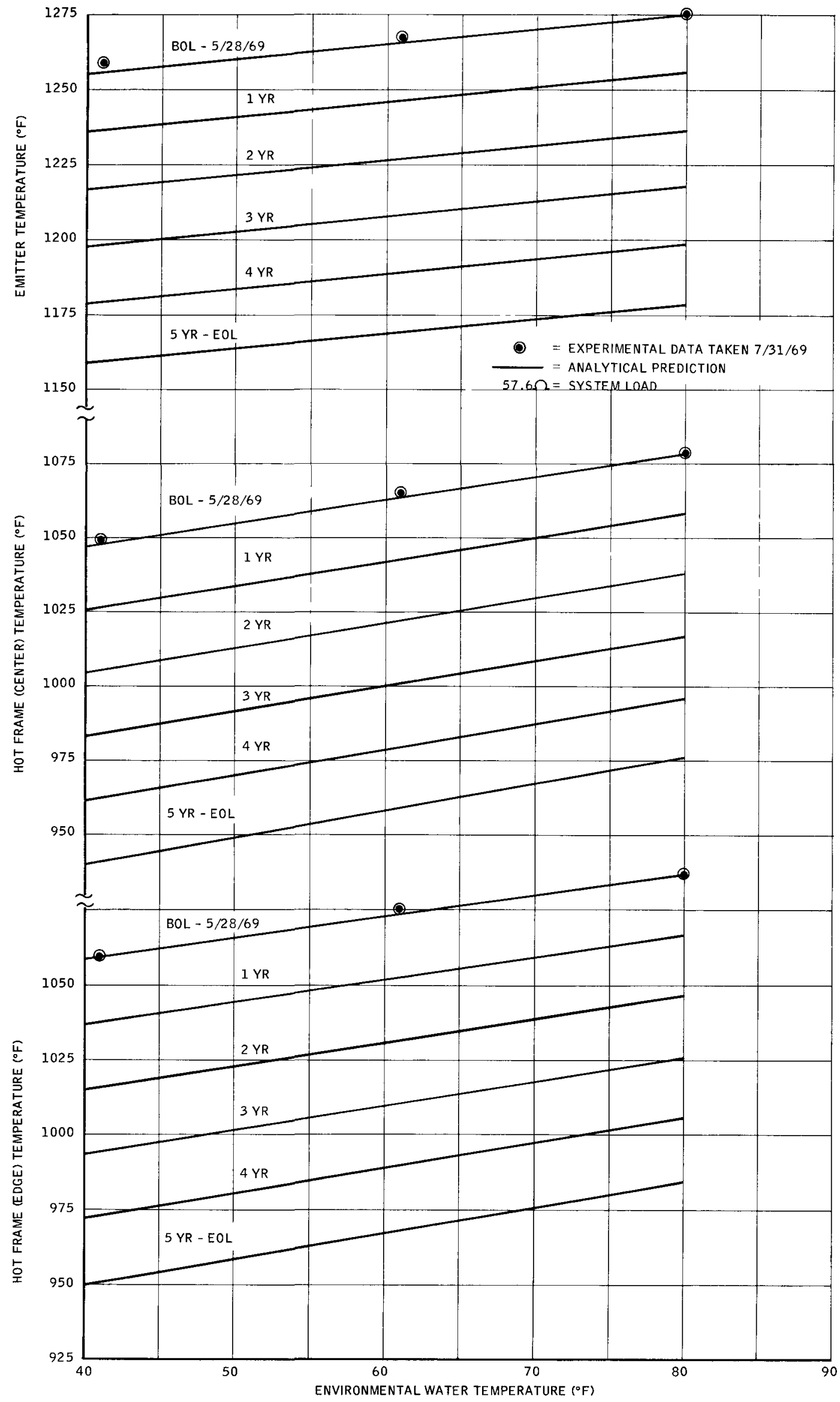

Figure 13. System S10P4 Performance System Temperatures vs. Water Temperatures 


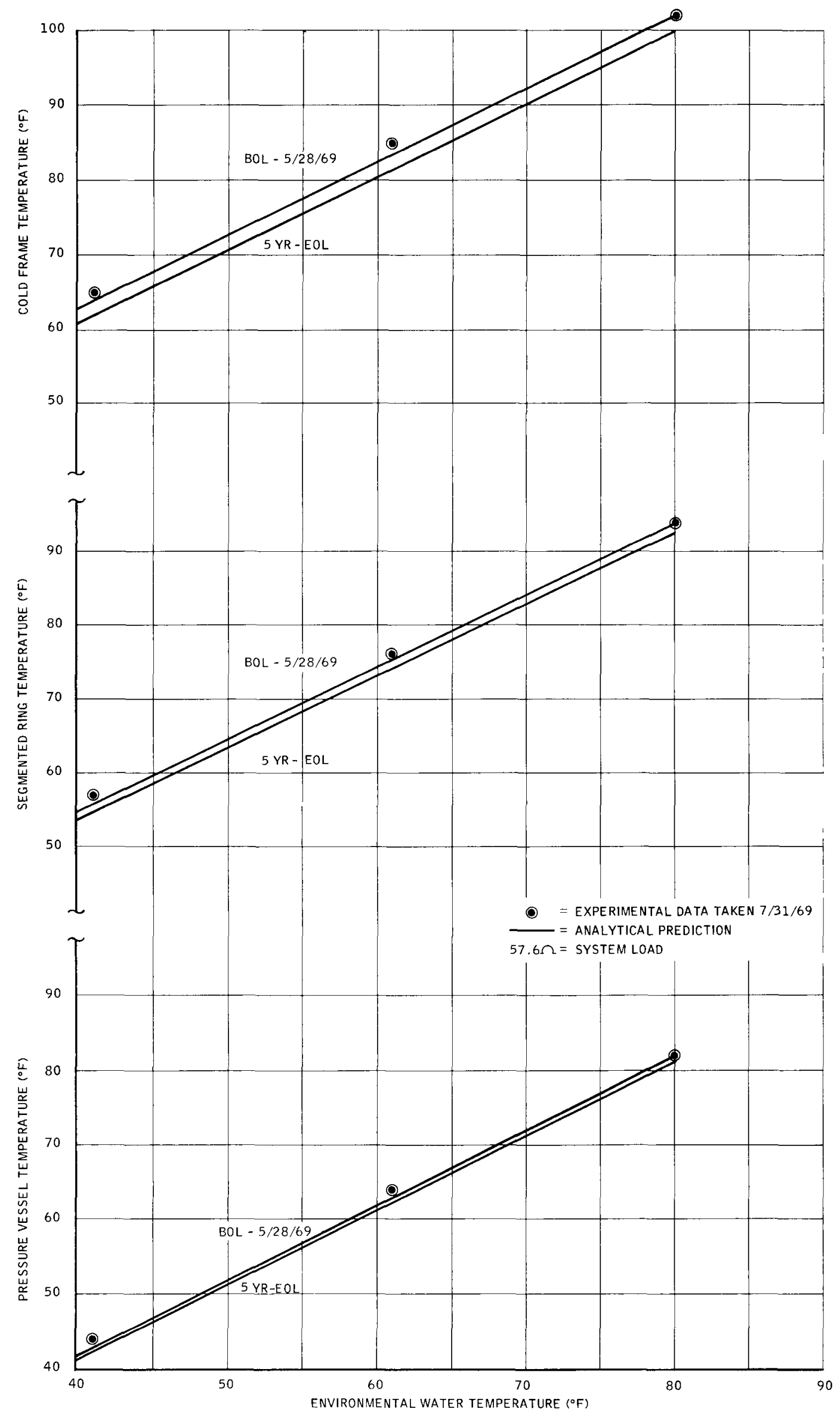

Figure 14. System S10P4 Performance System Temperatures vs. Water Temperatures 